\author{
Ahmet K. Firat \\ Hakki M. Karakas \\ Zeynep Y. Firat \\ Bayram Kahraman \\ Gülnur Erdem
}

\section{Spontaneous intracranial hypotension with pituitary adenoma}

Received: 5 December 2005

Accepted in revised form: 12 January 2006

Published online: 20 February 2006

\author{
A.K. Firat $(\square) \cdot$ H.M. Karakas \\ B. Kahraman • G. Erdem \\ Department of Radiology, \\ Inonu University Medical Faculty \\ Turgut Ozal Medical Center, \\ Malatya, Turkey \\ e-mail: akfirat@inonu.edu.tr \\ Tel.: +90-422-341-0660/5704 \\ Fax : +90-422-336-3084
}

\section{Z.Y. Firat}

Department of Otorhinolaryngeology, Inonu University Medical Faculty

Turgut Ozal Medical Center,

Malatya, Turkey

\begin{abstract}
Spontaneous intracranial hypotension $(\mathrm{SIH})$ is an unusual syndrome that is characterised by positional headache, neck rigidity, nausea and vomiting. The characteristic magnetic resonance imaging (MRI) findings are diffuse smooth pachymeningeal thickening and enhancement, downward displacement of posterior fossa structures and pituitary gland enlargement. An unusual case of SIH with pituitary macro-adenoma and subsequent subdural haemorrhage is presented, and its clinical picture, MRI findings and possible pathophysiological mechanism are discussed.
\end{abstract}

Keywords Spontaneous intracranial hypotension - Pituitary adenoma • Pachymeningeal thickening • Subdural haemorrhage

\section{Introduction}

Acquired intracranial hypotension is more frequent than spontaneous ones and they are usually due to a lumbar puncture, spinal anaesthesia and neurosurgical operations. Spontaneous intracranial hypotension (SIH) was first described by Schaltenbrand in 1938 [1]. SIH is a rare phenomenon characterised by postural headache, neck pain, nausea/vomiting and findings of cranial neuropathies. Although it was already a well known clinical entity, the diagnostic value of imaging was better valued after magnetic resonance imaging (MRI) became widely available. The characteristic MRI findings are diffuse intracranial pachymeningeal thickening and enhancement, downward displacement of posterior fossa structures and pituitary gland enlargement. However, SIH associated with pituitary adenoma has not been previously reported. In this paper, a SIH case with pituitary adenoma and associated subsequent subdural haemorrhage is presented.

\section{Case report}

A 41-year-old woman was presented to our hospital with a one-year history of severe headache, 


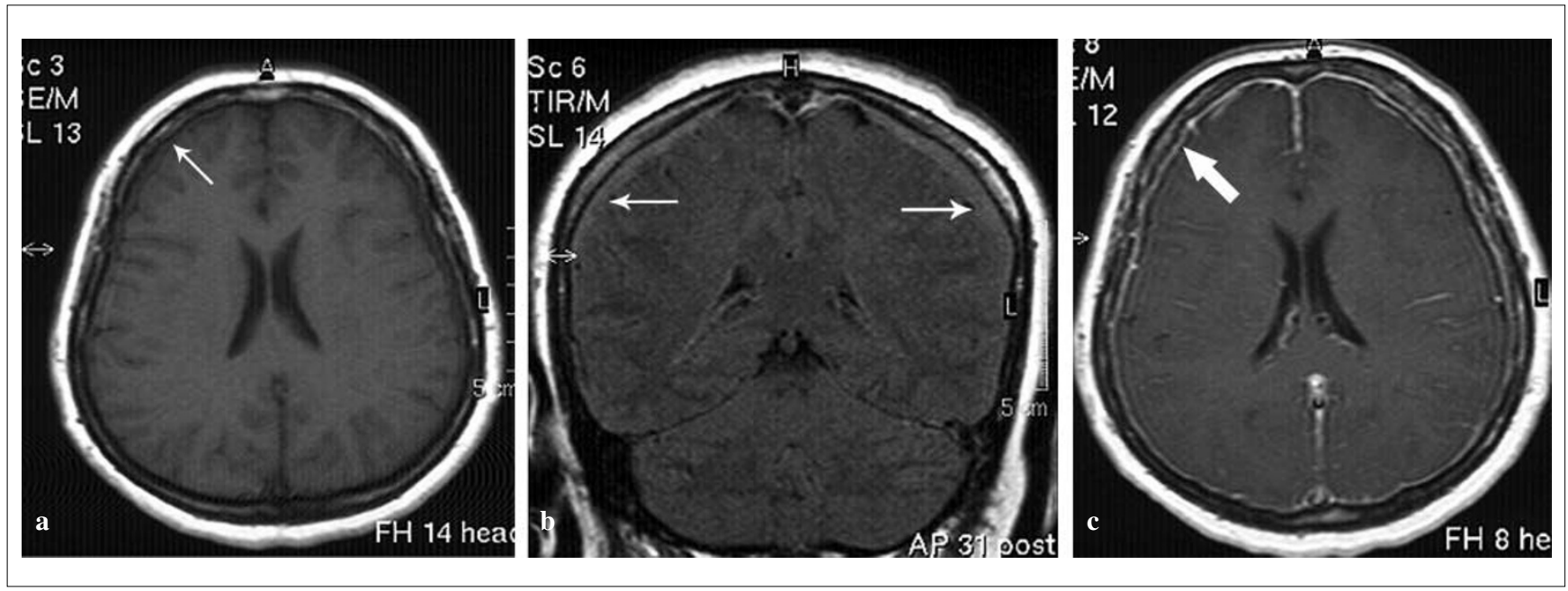

Fig. 1 a Axial T1-weighted MRI. The dura matter over cerebral convexities is isointense to cerebral cortex (arrow). b Coronal FLAIR image. The dura matter is relatively hyperintense (arrows). c Post-gadolinium axial T1-weighted image. Diffuse smooth dural thickening with enhancement and bilateral frontal subdural collections (arrows)

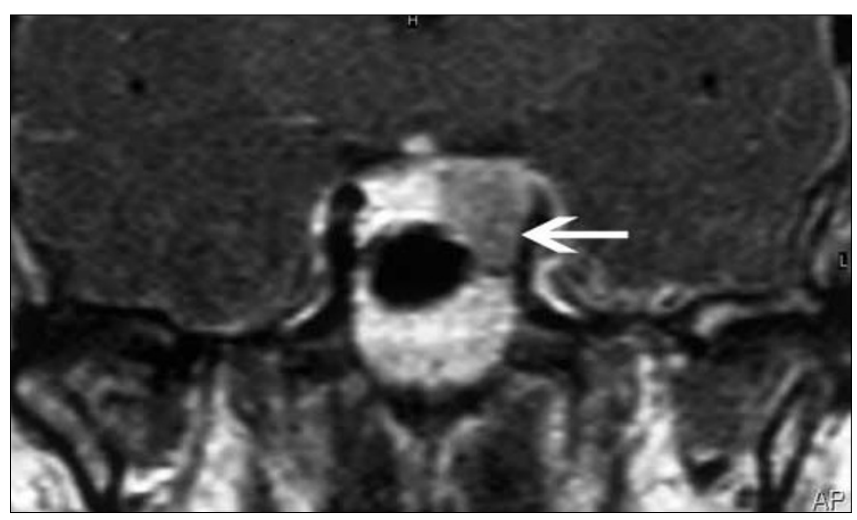

Fig. 2 Post-gadolinium coronal T1-weighted image: pituitary macro-adenoma (arrow) and accompanying adenohypophyseal deviation

nausea/vomiting and acoustic impairment. Whereas her headache had not responded to analgesics, it improved in the supine position. On neurological examination, neck rigidity, cranial nerve palsy and neurological deficits were absent. Her body temperature was $37.6^{\circ} \mathrm{C}$ and no sign of infection was noted. History of trauma or surgery that might initiate cerebrospinal fluid (CSF) leakage was not present.

On the cranial MRI performed in $1.5 \mathrm{~T}$ scanner, a diffuse smooth dural thickening of $3 \mathrm{~mm}$ was noted. Dural membranes were isointense to brain parenchyma on T1weighted, and relatively hyperintense on T2 and fluidattenuated inversion recovery (FLAIR) images. Intense dural enhancement and bilateral frontal subdural collections were also observed (Fig. 1). Cerebellar tonsils were situated normally, whereas pons was flattened, basal cisterns were narrowed and iter was descended on sagital images. Pituitary gland was asymmetrically larger and its maximum height was measured to be $10 \mathrm{~mm}$ in the midsagittal plane. Prominent cavernous sinuses were also observed.

A spinal MRI was acquired to investigate the possible site of CSF leakage. No evidence of Tarlov's cyst, dural tear or arachnoid diverticula was noted. As the pituitary gland was asymmetrically enlarged, a dynamic pituitary gland MRI was performed. On the left side, a pituitary

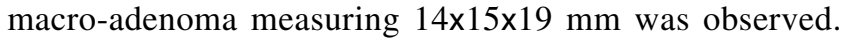
The mass was deviating the normal adenohypophysis to right side and indenting the left lateral side of the sellar floor (Fig. 2). Pituitary laboratory tests including T3, T4, TSH, FSH, LH, prolactin, progesterone and oestradiol were within normal ranges.

We recommended lumbar puncture and myelography for CSF pressure measurement and analysis, and also to demonstrate the site of CSF leakage. Some of the physicians opposed lumbar puncture as it might exacerbate the symptoms. They recommended a conservative treatment protocol including bed rest and oral hydration. Three months later, she presented with the same symptoms without significant change. On the MRI, meningeal enhancement and thickening were not modified. At the six-month examination, her symptoms were improved but had not totally disappeared. However on MRI, the meningeal enhancement and thickening were increased compared to previous images, and surprisingly a subsequent subacute subdural haemorrhage was noted (Fig. 3). As the patient refused to undergo further investigation and blood patch therapy, she was under our control without significant complaint except improving headache. 

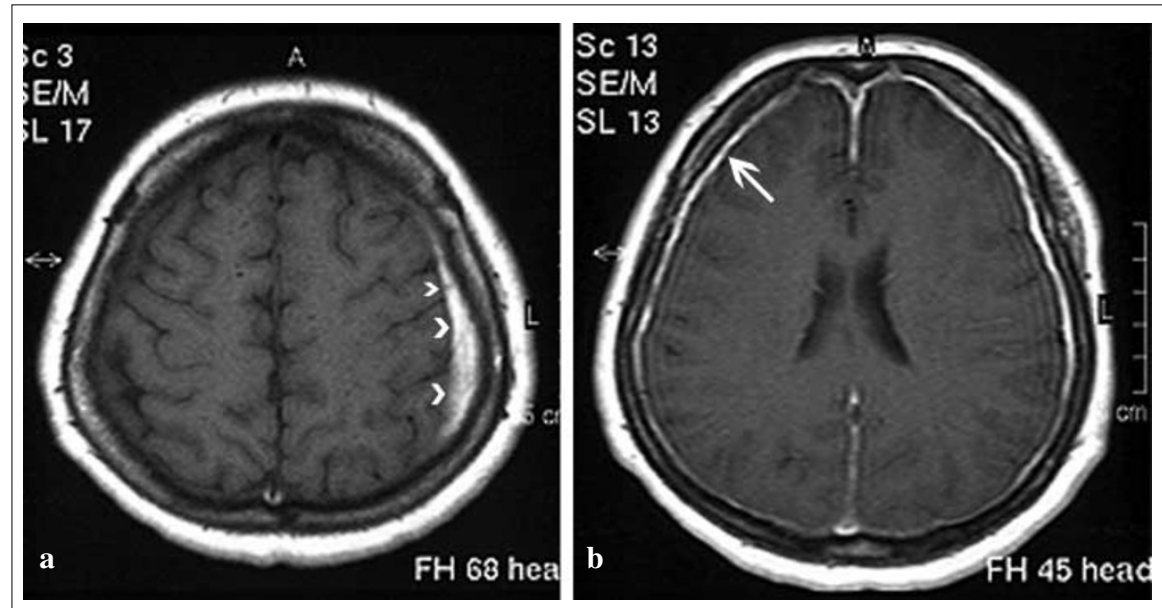

Fig. 3 Six-month follow-up. Pre- (a) and post-gadolinium (b) axial T1-weighted MRI revealed interval elevated thickening of dura (arrow) compared to previous MRI and subsequent subdural haemorrhage (arrowheads)

\section{Discussion}

Clinical features of SIH are usually pathognomonic. Postural headache unresponsive to analgesics is the main characteristic symptom. It is aggravated in the upright position and decreased in the recumbent position. Valsalva manoeuvre and head shaking may also aggravate the headache [2]. Nausea/vomiting, tinnitus, vertigo, photophobia, visual field defects, neck traction and cranial nerve palsies are among the other clinical features [2, 3].

CSF pressure evaluation is a reliable method for the diagnosis of SIH and the pressure is usually less than 5-6 $\mathrm{cm} \mathrm{H}_{2} \mathrm{O}$ [4, 5]. CSF analysis can be normal or it may reveal increased protein and white cell count [2-4, 6, 7]. It is not recommended anymore, as it may exacerbate the headache [6]. The presented case had features of abovementioned clinical and imaging findings; however it was especially remarkable for subsequent subdural haemorrhage. Although a conservative approach is advised in the relevant literature, lumbar puncture for CSF pressure measurement, radioisotope cisternography and CT myelography might be considered in long-acting or persistent disease.

The cardinal MRI finding of SIH is the continuous smooth thickening and diffuse intense enhancement of dura matter [3, 8]. Subdural collections, prominent cavernous sinus and increased pituitary gland height may also be seen [7]. The flattening of the pons, ventricles and basal cisterns, representing the downward displacement of the brain and brainstem, can be observed. These findings may be subtle when the patient is examined in the supine position, and they may therefore easily be missed [8]. As a late manifestation, descent of cerebellar tonsils may be noted [2]. On spinal MRI, meningeal diverticula or dural tears may be observed [2]. Diffuse dural enhancement of the spinal canal was also described [3]. In the presented case, with the exception of dural tear, meningeal divertucula and spinal dural enhancement, the classical neuroradiological findings were observed.

Diffuse dural enhancement and prominent cavernous sinus represent the venous congestion that occurs to compensate the intracranial volume loss in accordance with the Monroe-Kellie doctrine [9].

Transient pituitary gland enlargement is a rare but interesting MRI finding in SIH. In such cases an upward bowing of the diaphragma sella, and an increase in pituitary height in the mid-sagittal plane may be observed [3, 10]. These findings are transient and they disappear on subsequent follow-ups. They are also thought to be caused by venous engorgement, however the mechanism is not very clear [3]. The presence of pituitary adenoma, if not coincidental, may change the above-mentioned hypothesis explaining the pituitary enlargement. In that situation, one might speculate that a disease with short duration with proper treatment may have transient causes; whereas a chronic course may end up with over-stimulation, causing a nonfunctioning pituitary adenoma. This over-stimulation may be due to altered neural regulation and feedback control of the hypothalamic-pituitary axis.

Our patient also exhibited the above-mentioned MRI findings: upward bowing of the diaphragma sella and increase in pituitary height in the mid-sagittal plane. In addition to those, she also had a pituitary macro-adenoma. That association had not been reported before and it was thought to be either coincidental or related. Still, this association reminded us of the utility of a dedicated pituitary MRI in this disease, as an underlying pituitary pathology may be masked by the pituitary changes. The appropriate time for scheduling is probably the follow-up period, after resolution of pituitary enlargement.

We observed persistent meningeal thickening and enhancement in the subsequent MRI examinations of the presented case. According to us, this finding pointed out 
the ineffectiveness of the treatment protocol and the need for further evaluation and therapy. The appearance of subdural haemorrhage at the follow-up supported our proposal. In this situation, the risk of clinical exacerbation due to lumbar puncture and blood patching should be accepted in order to avoid mortality and morbidity due to subdural haemorrhage. The meningeal thickening can be used as an additional and independent prognostic factor in SIH.

In conclusion, the availability of MRI has greatly influenced the diagnosis of SIH. Although the diagnosis is usually based on clinical and laboratory investigations, imaging is necessary for the differentiation of SIH from other life-threatening intracranial pathologies and also for grading of disease. However, the imaging features of SIH may be misleading because of their similarity to the features of other disease, such as hypertrophic pachymeningitis, granuloumatous and malignant dural involvement, and pituitary pathologies. Therefore, the clinician should be aware of the necessity of further diagnostic work-up.

\section{References}

1. Schaltenbrand G (1938) Neuere Anschauungen zur Pathophysiologie der Liquorzirkulation. Zentralbl Neurochir 3:400-413

2. Rondo TA, Fishman RA (1992) Spontaneous intracranial hypotension: report of two cases and review of the literature. Neurology 42:481-487

3. Tsui EYK, Ng SH, Cheung YK, Fong D, Yuen MK (2001) Spontaneous intracranial hypotension with dural enhancement of the spinal canal and transient enlargement of the pituitary gland. Eur J Radiol 38:59-63
4. Mokri B (1999) Spontaneous cerebrospinal fluid leak; from intracranial hypotension to cerebrospinal fluid hypovolemia evaluation of a concept. Mayo Clin Proc 74:1113-1123

5. Ferrante E, Savino A, Sances G, Nappi G (2004) Spontaneous intracranial hypotension syndrome: report of twelve cases. Headache 44:615-622

6. Fishman RA, Dillon WP (1997) Intracranial hypotension. J Neurosurg 86:165

7. Mokri B, Piepgras DG, Miller MG (1997) Syndrome of orthostatic headaches and diffuse pachymeningeal gadolinium enhancement. Mayo Clin Proc 72:400-413
8. Metafratzi Z, Argyropoulou MI, Mokou-Kanta C, Konitsiotis S, Zikou A, Efremidis SC (2004) Spontaneous intracranial hypotension: morphological findings and CSF flow dynamics studied by MRI. Eur Radiol 14:1013-1016

9. Mokri B (2001) The Monro-Kellie hypothesis: application in CSF volume depletion. Neurology 56:1746-1748

10. Tapia DQ, Pardo J, Serrano JM, Anciones V, Lopez-Ibor L (2000) Spontaneous intracranial hypotension: use of unenhanced MRI.

Neuroradiology 42:529-531 